\title{
COMPOSICIÓN NUTRIMENTAL DEL NOPAL VERDURA PRODUCIDO CON FERTILIZACIÓN MINERAL Y ORGÁNICA
}

\author{
NUTRITIONAL COMPOSITION OF TENDER CACTUS STEMS \\ PRODUCED UNDER MINERAL AND ORGANIC FERTILIZATION
}

\author{
Ma. del Rocío Santiago-Lorenzo, Alfredo López-Jiménez*, Crescenciano Saucedo- \\ Veloz, José I. Cortés-Flores, David Jaén-Contreras y Javier Suárez-Espinosa
}

Recursos Genéticos y Productividad-Fruticultura, Campus Montecillo, Colegio de Postgraduados. Km. 36.5 carretera México-Texcoco. 56230, Montecillo, Texcoco, Estado de México. Tel.: (595) 9520200 Ext. 1116.

*Autor para correspondencia (lopezja@colpos.mx)

\section{RESUMEN}

La calidad nutritiva del nopal verdura, está relacionada con su composición mineral y es variable debido a varios factores, uno de ellos es fertilización. En nopal verdura (Opuntia ficus-indica (L.) Mill.) la respuesta a la aplicación de fertilizantes orgánicos e inorgánicos se ha enfocado principalmente al rendimiento y en menor proporción con la calidad nutricional. El objetivo fue conocer el efecto de la fertilización mineral y orgánica incluyendo el uso de compost y la inoculación con micorriza en la composición mineral de cladodios jóvenes para consumo humano al momento de cosecha. Los tratamientos estudiados fueron: 1) Estiércol bovino más fertilizante 17N-17P-17K (E + T 17); 2) Compost más inoculación con el hongo micorrícico Glomus mosseae $(\mathrm{C}+\mathrm{M})$; 3 ) Fertilizantes minerales (FM), aplicados por fertirriego; y 4) Testigo, sin aplicación de fertilizante y sin micorriza. El riego por goteo fue aplicado una vez por semana o cuando la tensión de humedad en el suelo llegaba a $35 \mathrm{cbar}$. El tratamiento de fertilización tuvo un efecto significativo en las concentraciones de Mn, Zn y B, no así en N, P, K, Ca, Mg, Fe y Cu en el cladodio joven o nopalito. La más alta concentración de Mn (81.38 $\mathrm{mg} \mathrm{kg}^{-1}$ ) se alcanzó con el tratamiento FM, la de Zn cuando se aplicó $\mathrm{E}+\mathrm{T} 17$ (44.11 $\left.\mathrm{mg} \mathrm{kg}^{-1}\right)$, y en el caso de B con la fertilización de FM y $\mathrm{E}+\mathrm{T} 17\left(156.09\right.$ y $\left.151.77 \mathrm{mg} \mathrm{kg}^{-1}\right)$, respectivamente.

Palabras clave: Opuntia ficus indica, composición mineral, fertilización orgánica, fertirriego, fósforo disponible, zinc.

\section{SUMMARY}

The nutritional quality of tender cactus stem is related to its mineral composition, which is variable due to several factors. Fertilization source is one of them. In tender cactus stems (Opuntia ficus-indica (L.) Mill.), response to application of organic and inorganic fertilizers are mainly focused on yield and to a lesser extent to the nutritional quality. The objective was to determine the effect of mineral and organic fertilization including the use of compost and inoculation with mycorrhiza on mineral composition of tender cactus stem harvested for human consumption. The studied treatments were: 1) Cow manure plus 17N-17P-17K fertilizer (E + T 17); 2) Compost and Glomus mosseae mycorrhizal fungi $(\mathrm{C}+\mathrm{M})$; 3 ) Mineral fertilizers (FM); and 4) Control, no fertilizer, compost or mycorrhizal fungi added. Drip irrigation was applied once a week or when the soil moisture tension reached 35 cbar. Fertilization source had a significant effect on the concentrations of $\mathrm{Mn}, \mathrm{Zn}$ and $\mathrm{B}$ but not on $\mathrm{N}, \mathrm{P}, \mathrm{K}, \mathrm{Ca}, \mathrm{Mg}$, Fe and $\mathrm{Cu}$ in dry matter tender of cactus stem. The highest concentration of $\mathrm{Mn}\left(81.38 \mathrm{mg} \mathrm{kg}^{-1}\right)$ was achieved with the FM treatment. Highest content of $\mathrm{Zn}\left(44.11 \mathrm{mg} \mathrm{kg}^{-1}\right)$ was reached with the fertilization of $E$ $+T$ 17, and for $B\left(156.09\right.$ and $\left.151.77 \mathrm{mg} \mathrm{kg}^{-1}\right)$ with $F M$ and $E+T 17$ respectively.

Index words: Opuntia ficus-indica, fertirrigation, mineral composition, organic fertilization, available phosphorus, zinc.

\section{INTRODUCCIÓN}

El consumo de nopal verdura (Opuntia ficus-indica L. Mill.) se ha incrementado en los últimos años, debido a las propiedades nutracéuticas que se le atribuyen. México produjo 824,602 t en 2014 y 723,815 t en 2010 y es el principal país productor de esta hortaliza y el mayor consumidor a nivel mundial, con un consumo per capita de $6.7 \mathrm{~kg}$ (SIAP, 2014). Países como Estados Unidos, Canadá, Japón, Italia y Turquía demandan el nopal verdura debido a su bajo valor calórico, alto contenido en fibra y cualidades nutricionales y funcionales (Sáenz-H., 2004; Stintzing y Carle, 2005). Estudios realizados en humanos lo colocan como un alimento funcional para tratar la enfermedad de la diabetes por su riqueza de fibra dietética (Sloan, 1994; Sáenz-H., 1997; Torres y Torres, 2014).

La calidad nutritiva del nopal verdura está relacionada con su composición mineral, la cual es variable debido a varios factores. Zhao y Shewry (2011) plantean tres estrategias para aumentar la cantidad de elementos minerales en las partes comestibles de las plantas: el mejoramiento genético convencional, la biotecnología y el manejo agronómico; con relación a esta última, la fertilización constituye una de las principales propuestas. En los huertos de nopal para tuna, el método de fertilización más usado es la aplicación al suelo de abonos orgánicos, fertilizantes químicos o la combinación de ambos (Pimienta-Barrios, 1990; Murillo-Amador et al., 2005).

En plantaciones convencionales de nopal verdura como 
las existentes en Milpa Alta, México, la fertilización se hace entre hileras con una capa de 10 a $15 \mathrm{~cm}$ de estiércol, generalmente de bovino, cada dos o tres años. Algunos productores también aplican urea o sulfato de amonio de una a tres veces al año. En sistemas intensivos se aplican de 100 a 200 t de abono orgánico por ha, y adicionalmente de 100 a $200 \mathrm{~kg} \mathrm{ha}^{-1}$ de nitrógeno y de 80 a $100 \mathrm{~kg} \mathrm{ha}^{-1} \mathrm{de}$ fósforo (Flores-Valdez, 2003). En un estudio realizado en Opuntia ficus-indica (L.) Mill., Zúñiga-Tarango et al. (2009) encontraron que aplicaciones de $100 \mathrm{t} \mathrm{ha}^{-1}$ de estiércol de bovino ocasionaron en los cladodios tiernos de consumo una mayor concentración de Mn, Cu, Zn y Fe (39.6, 18.9, 44.8, y 179.7 ppm, respectivamente), de P (0.56 \%) y K (6.58 $\%)$, y en el suelo un incremento en el contenido mineral y de materia orgánica.

Fernández-Pavía et al. (2015) registraron concentraciones promedio de $2.2,0.8,5.7,3.3$ y $1.7 \%$ de ms para $\mathrm{N}, \mathrm{P}$, $\mathrm{K}, \mathrm{Ca}, \mathrm{y} \mathrm{Mg}$, respectivamente, en nopalitos de la var. Milpa Alta de 25 a $30 \mathrm{~cm}$ de longitud y $13 \mathrm{~cm}$ de ancho, cuando se fertilizaron con una solución nutritiva completa. La concentración de nutrimentos en cladodios de Nopalea gaumeri Britton \& Rose está en el orden de 81,20 y $2.3 \mathrm{mg}$ por $100 \mathrm{~g}$ ms para Ca, P y Fe, respectivamente (BolívarFernández et al., 2009).

La inoculación con micorrizas y la aplicación de compost en el nopal verdura han sido poco estudiadas y la mayoría de los trabajos de fertilización en nopal verdura se han centrado en el rendimiento, y en menor proporción sobre la composición mineral del cladodio de tamaño comercial, y en su relación con el aporte de minerales para el consumo humano. El objetivo del trabajo fue evaluar el efecto de la fertilización mineral y orgánica incluyendo el uso de compost y la inoculación con micorriza en la composición mineral de cladodios jóvenes o nopalitos para consumo humano al momento de cosecha.

\section{MATERIALES Y MÉTODOS}

El experimento se hizo con nopal verdura de la variedad Milpa Alta de cuatro años de edad, en condiciones de campo en Montecillo, Texcoco, Estado de México (19² $28^{\prime} \mathrm{N}$ y $98^{\circ} 47^{\prime}$ O). El clima del sitio es templado-cálido con lluvias en verano, con precipitación anual promedio de $772 \mathrm{~mm}$; la temperatura máxima mensual osciló entre 28 y $33.5^{\circ} \mathrm{C}$ durante el periodo de mayor brotación de nopalitos (mayojunio), en tanto que la temperatura mínima varió de 15 a $17^{\circ} \mathrm{C}$.

El experimento se estableció en 2010 y en 2013 se hizo la evaluación la cual incluyó los tratamientos: 1) Estiércol de bovino más fertilizante triple 17 (E + T 17); 2) Compost más inoculación con el hongo Glomus mosseae $(C+M)$;
3) Fertilizantes minerales (FM), que se detallan abajo; y 4) Testigo, sin aplicación de fertilizante y sin micorriza. El estiércol de bovino y triple 17 se aplicaron en el mes de enero de 2013 a una dosis de $5 \mathrm{~kg}$ y $80 \mathrm{~g}$ por planta, respectivamente; el compost se aplicó a una dosis de $5 \mathrm{~kg}$ por planta; y la inoculación con el hongo micorrícico se hizo con $20 \mathrm{~g}$ de sustrato de raíces de bálsamo (Impatiens balfourii). El compost y el hongo fueron aplicados en la superficie del suelo en banda en el mes de agosto de 2012.

Los fertilizantes minerales (FM) fueron: multi NPK (13N0.86P-36.7K), fosfato monoamónico (12N-26P-0K), nitrato de calcio (15.5N-OP-OK-18Ca), sulfato de potasio (ON-OP$36 \mathrm{~K}-18 \mathrm{~S})$, y superfosfato de calcio triple (ON-20P-0K), a dosis de 34, 7, 6 y $46 \mathrm{~g}$ por planta por año, respectivamente. La dosis se fraccionó y tres partes se aplicaron en mayo y tres partes en agosto de 2013, vía fertirriego. Todos los tratamientos tuvieron riego por goteo una vez por semana o cuando la tensión de humedad del suelo alcanzaba 35 cbar, con una lámina mensual de $33 \mathrm{~mm}$.

Se utilizó un diseño experimental de bloques al azar con tres repeticiones. La unidad experimental fue de cinco plantas por tratamiento, con las tres plantas centrales como parcela útil. En enero de 2013 se muestreó el suelo de cada tratamiento a una profundidad de 0 a $30 \mathrm{~cm}$ para su análisis químico. La materia orgánica $(\mathrm{MO})$ se determinó por el método de Walkley y Black (León-Arteta y AguilarSantelises, 1987), la concentración de P por el método de Olsen, los elementos Fe, Cu, Zn, Mn, B fueron extraídos por DTPA $0.005 \mathrm{M}$ y cuantificados por absorción atómica en un espectrofotómetro Varian® SpectrAA 220FS (Australia). El contenido de $\mathrm{N}$ total fue estimado tras multiplicar el valor de MO por el factor 0.05 (Castellanos et al., 2000).

Para determinar la composición mineral de los nopalitos, en el mes de junio de 2013 de cada unidad experimental se obtuvo una muestra de tres cladodios tiernos (nopalitos) de 20 a $25 \mathrm{~cm}$ de longitud. Los cladodios fueron lavados, desespinados y fraccionados. Posteriormente fueron secados a $70{ }^{\circ} \mathrm{C}$ en una estufa Imperial V® (Iowa, USA) durante $48 \mathrm{~h}$, hasta obtener peso constante. La molienda se hizo con un molino de acero inoxidable General Electric ${ }^{\circledR}$ (Modelo AC 160, California, USA) con tamiz de 2 mm.

La determinación de $\mathrm{N}$ se realizó por el método MicroKjeldahl (Bremner, 1965). Los elementos P, K, Ca, Mg, Fe, Mn, $\mathrm{Zn}$, Cu y $\mathrm{B}$ se determinaron en el filtrado del extracto proveniente de la digestión de cada muestra. La concentración de $\mathrm{K}, \mathrm{Ca}, \mathrm{Mg}, \mathrm{Fe}, \mathrm{Mn}, \mathrm{Zn}$, Cu y B se cuantificó por medio de un espectrómetro de emisión atómica (AES-ICP) marca Varian ${ }^{\circledR}$ modelo 725-ES (Mulgrave, Australia), y la concentración de P se midió por colorimetría del complejo fosfovanadomolibdato, de acuerdo con el método descrito por 
la AOAC (1980), con las lecturas respectivas a $470 \mathrm{~nm}$ en un espectrofotómetro Milton Roy ${ }^{\circledR}$ modelo Spectronic 20 (USA)

Los datos fueron sujetos a análisis de varianza, y la separación de medias se hizo mediante la prueba de Tukey $(a=0.05)$, con el paquete estadístico SAS versión 9.0 (SAS, 2000).

\section{RESULTADOS Y DISCUSIÓN}

\section{Composición química del suelo}

Los contenidos de N, P y Mn en el suelo fueron afectados significativamente $(P \leq 0.05)$ por el tratamiento de fertilización; $\mathrm{E}+\mathrm{T} 17$ y C + M fueron superiores en N (0.2 y $0.2 \%$, respectivamente), $\mathrm{E}+\mathrm{T} 17$ en $\mathrm{P}\left(107.5 \mathrm{mg} \mathrm{kg}^{-1}\right)$, y $\mathrm{C}+\mathrm{M}$ en $\mathrm{Mn}$ (4.1 $\mathrm{mg} \mathrm{kg}^{-1}$ ) (Cuadro 1). Estos resultados permiten inferir que la aplicación sucesiva de estiércol mejoró la fertilidad del suelo particularmente en los nutrimentos $\mathrm{N}$ y $\mathrm{P}$, aun cuando el contenido de materia orgánica (MO) no fue estadísticamente diferente entre tratamientos.

Los contenidos de $\mathrm{MO}$ con todos los tratamientos estuvieron en el intervalo de medio a alto, los de $\mathrm{N}$ de moderadamente pobre a medio, los de P, K, Ca y Mg alto, en Mn y en $\mathrm{Cu}$ bajo, de muy bajo a bajo en Fe, de moderadamente bajo a medio en $\mathrm{Zn}$, y de moderadamente bajo a moderadamente alto en $\mathrm{B}$, de acuerdo con los valores de referencia reportados por Castellanos et al. (2000). Por otra parte, el bajo contenido de $\mathrm{Cu}, \mathrm{Zn}$, Fe y $\mathrm{Mn}$ obedece al pH alcalino del suelo, que fue de 7.9 a 8.3.

\section{Composición nutrimental del nopal verdura}

La composición mineral de los nopalitos var. Milpa Alta no fue diferente $(\mathrm{P} \leq 0.05)$ en $\mathrm{N}, \mathrm{P}, \mathrm{K}, \mathrm{Ca}, \mathrm{Mg}$, Fe y $\mathrm{Cu}$, pero sí en Mn, Zn y B (Cuadros 2 y 3 ). La falta de respuesta a los elementos mayores es similar a la reportada por ZúñigaTarango et al. (2009) al estudiar el efecto de la aplicación de estiércol bovino en dosis de 100 y $300 \mathrm{t} \mathrm{ha}^{-1}$, ya sea solo estiércol o en combinación con fertilizante mineral. De acuerdo con estos investigadores, el resultado final depende de las interacciones entre los elementos en el suelo durante la absorción de los mismos por las raíces.

Según Bould (1970), la absorción de un ion en la planta debe ser considerada en función de su concentración individual y de su interacción con los demás iones, y no en forma aislada. Con los fertilizantes minerales la concentración de $\mathrm{N}$ fue de $1.7 \%$, y con los tratamientos de estiércol bovino + triple 17 y en $\mathrm{C}+\mathrm{M}$ fueron de 1.6 y $1.2 \%$ de $\mathrm{ms}$, respectivamente (Cuadro 2). Con la aplicación de estiércol de bovino + triple 17 se alcanzó una concentración de P de $0.54 \%$ de ms, similar a las reportadas por Zúñiga-Tarango et al. (2009) de 0.56 y $0.59 \%$ de P con aplicaciones de estiércol bovino en dosis de 100 y $300 \mathrm{t} \mathrm{ha}^{-1}$, respectivamente. Respecto al K, la concentración de 4.28 \% obtenida con compost y micorriza es superior al resto de tratamientos.

Con relación a los micronutrimentos, el cladodio de las plantas fertilizadas con FM presentó la más alta concentración de $\mathrm{Mn}$ (81.38 $\left.\mathrm{mg} \mathrm{kg}^{-1}\right)$, en tanto que la menor concentración fue con el compost y micorriza (Cuadro 3). En Zn solo hubo diferencia estadística entre los tratamientos $\mathrm{E}+\mathrm{T} 17$ y FM (44.1 vs. $9.9 \mathrm{mg} \mathrm{kg}^{-1}$ ), lo que sugiere que con el primer tratamiento se alcanza un aporte de $\mathrm{Zn}$ superior al de hortalizas de hoja (< $\left.10 \mathrm{mg} \mathrm{kg}^{-1}\right)$ (FAO/WHO, 2001).

El estiércol más fertilizante triple 17 se aplicó en el mes de enero y el tratamiento FM se aplicó más tarde durante los meses de mayo y agosto. Por tanto, en el primer tratamiento la planta tuvo más tiempo para absorber el Zn disponible en el suelo estimulado por el estiércol y el fertilizante. En B hubo diferencia entre FM y C + M (156.09 y $137.64 \mathrm{mg} \mathrm{kg}^{-1}$ ) (Cuadro 3).

Cuadro 1. Análisis químico del suelo a la profundidad de 0 a $30 \mathrm{~cm}$ de cada tratamiento de fertilización.

\begin{tabular}{|c|c|c|c|c|c|c|c|c|c|c|c|}
\hline \multirow{2}{*}{$\begin{array}{l}\text { Tratamiento } \\
\text { de fertilización }{ }^{\dagger}\end{array}$} & $\mathrm{MO}$ & $\mathrm{N}$ & \multirow{2}{*}{$\frac{P}{\mathrm{mg} \mathrm{kg}^{-1}}$} & $\mathrm{~K}$ & $\mathrm{Ca}$ & $\mathrm{Mg}$ & $\mathrm{Fe}$ & $\mathrm{Cu}$ & $\mathrm{Zn}$ & $\mathrm{Mn}$ & $\mathrm{B}$ \\
\hline & \multicolumn{2}{|c|}{$\%$} & & \multicolumn{3}{|c|}{ Meq / 100 g de suelo } & \multicolumn{5}{|c|}{$\mathrm{mg} \mathrm{kg}^{-1}$} \\
\hline$E+T 17$ & $3.5 \mathrm{a}$ & $0.2 \mathrm{a}$ & $107.5 \mathrm{a}$ & $1.6 \mathrm{a}$ & $14.4 \mathrm{a}$ & $4.2 \mathrm{a}$ & $6.1 \mathrm{a}$ & $0.3 a$ & $1.7 \mathrm{a}$ & $3.0 \mathrm{~b}$ & $1.1 \mathrm{a}$ \\
\hline$C+M$ & $3.2 \mathrm{a}$ & $0.2 \mathrm{a}$ & $30.2 b c$ & $1.2 \mathrm{a}$ & $12.0 \mathrm{a}$ & $4.2 \mathrm{a}$ & $4.6 \mathrm{a}$ & $0.4 a$ & $1.8 \mathrm{a}$ & $4.2 \mathrm{a}$ & $1.0 \mathrm{a}$ \\
\hline FM & $2.4 \mathrm{a}$ & $0.1 b$ & $21.3 c$ & $1.8 \mathrm{a}$ & $17.7 \mathrm{a}$ & $4.2 \mathrm{a}$ & $2.8 \mathrm{a}$ & $0.2 \mathrm{a}$ & $1.0 \mathrm{a}$ & $3.3 b$ & $0.7 \mathrm{a}$ \\
\hline Testigo & $2.3 \mathrm{a}$ & $0.1 b$ & $41.1 \mathrm{~b}$ & $1.5 \mathrm{a}$ & $15.7 \mathrm{a}$ & $4.8 \mathrm{a}$ & $2.3 \mathrm{a}$ & $0.2 \mathrm{a}$ & $1.3 \mathrm{a}$ & $2.3 c$ & $1.0 \mathrm{a}$ \\
\hline CV (\%) & 16.7 & 13.0 & 12.0 & 16.8 & 27.5 & 12.1 & 39.5 & 29.0 & 20.7 & 6.8 & $24.6 \mathrm{a}$ \\
\hline DMS (0.05) & 1.3 & 0.05 & 13.7 & 0.7 & 11.5 & 1.6 & 4.4 & 0.2 & 0.8 & 0.6 & 0.6 \\
\hline
\end{tabular}

Medias con letras iguales no son estadísticamente diferentes (Tukey, 0.05$) .{ }^{\dagger} \mathrm{E}+\mathrm{T} 17$ = estiércol de bovino más fertilizante triple $17 ; \mathrm{C}+\mathrm{M}=\mathrm{compost}$ más inoculación con el hongo micorrícico Glomus mosseae; FM = fertilizantes minerales; CV = coeficiente de variación; DMS = diferencia mínima significativa; $\mathrm{MO}=$ Materia orgánica. 
Cuadro 2. Efecto del tratamiento de fertilización en la concentración (\% de ms) de los macronutrimentos en el tejido de nopal verdura (Opuntia ficus-indica (L.) Mill.) var. Milpa Alta.

\begin{tabular}{lccccc}
\hline Tratamiento de fertilización $^{+}$ & $\mathrm{N}$ & $\mathrm{P}$ & $\mathrm{K}$ & $\mathrm{Ca}$ & $\mathrm{Mg}$ \\
\hline $\mathrm{E}+\mathrm{T} 17$ & $1.61 \mathrm{a}$ & $0.54 \mathrm{a}$ & $3.30 \mathrm{a}$ & $2.44 \mathrm{a}$ & $2.35 \mathrm{a}$ \\
$\mathrm{C}+\mathrm{M}$ & $1.26 \mathrm{a}$ & $0.48 \mathrm{a}$ & $4.28 \mathrm{a}$ & $2.38 \mathrm{a}$ & $2.55 \mathrm{a}$ \\
$\mathrm{FM}$ & $1.75 \mathrm{a}$ & $0.39 \mathrm{a}$ & $2.88 \mathrm{a}$ & $2.62 \mathrm{a}$ & $2.81 \mathrm{a}$ \\
Testigo & $1.40 \mathrm{a}$ & $0.43 \mathrm{a}$ & $3.22 \mathrm{a}$ & $2.88 \mathrm{a}$ & $2.81 \mathrm{a}$ \\
$\mathrm{CV}$ & 13.69 & 8.32 & 10.48 & 4.47 & 4.27 \\
DMS (0.05) & 0.99 & 0.18 & 1.73 & 0.55 & 0.54 \\
\hline
\end{tabular}

Medias con letras iguales no son estadísticamente diferentes (Tukey, 0.05$) .{ }^{\dagger} \mathrm{E}+\mathrm{T} 17$ = estiércol de bovino más fertilizante triple 17; $C+M=$ compost más inoculación con el hongo micorrícico Glomus mosseae; FM = fertilizantes minerales; $\mathrm{CV}=$ coeficiente de variación; DMS = diferencia mínima significativa.

Cuadro 3. Efecto del tratamiento de fertilización en la concentración ( $\mathrm{mg} \mathrm{kg}^{-1}$ de $\mathrm{ms}$ ) de micronutrimentos en el tejido de nopal verdura (Opuntia ficus-indica (L.) Mill.) var. Milpa Alta.

\begin{tabular}{lccccc}
\hline Tratamiento de fertilización $^{+}$ & $\mathrm{Fe}$ & $\mathrm{Mn}$ & $\mathrm{Zn}$ & $\mathrm{Cu}$ & $\mathrm{B}$ \\
\hline $\mathrm{E}+\mathrm{T} 17$ & $59.89 \mathrm{a}$ & $45.00 \mathrm{~b}$ & $44.11 \mathrm{a}$ & $9.63 \mathrm{a}$ & $151.77 \mathrm{ab}$ \\
$\mathrm{C}+\mathrm{M}$ & $69.03 \mathrm{a}$ & $30.52 \mathrm{c}$ & $26.31 \mathrm{ab}$ & $9.18 \mathrm{a}$ & $137.64 \mathrm{c}$ \\
FM & $57.21 \mathrm{a}$ & $81.38 \mathrm{a}$ & $9.91 \mathrm{~b}$ & $7.65 \mathrm{a}$ & $156.09 \mathrm{a}$ \\
Testigo & $68.91 \mathrm{a}$ & $46.44 \mathrm{~b}$ & $27.85 \mathrm{ab}$ & $7.15 \mathrm{a}$ & $143.19 \mathrm{~b}$ \\
CV & 9.20 & 3.49 & 18.53 & 6.68 & 2.99 \\
DMS (0.05) & 28.3 & 5.01 & 24.19 & 2.71 & 12.44 \\
\hline
\end{tabular}

Medias con letras iguales no son estadísticamente diferentes (Tukey, 0.05). ${ }^{\dagger} E+T 17$ = estiércol de bovino más fertilizante triple 17; $\mathrm{C}+\mathrm{M}=$ compost más inoculación con el hongo micorrícico Glomus mosseae; $\mathrm{FM}=$ fertilizantes minerales; $\mathrm{CV}=$ coeficiente de variación; DMS = diferencia mínima significativa.

Zúñiga-Tarango et al. (2009) reportaron concentraciones (\% de ms) de 6.2, 5.4 y 1.0 para K, Ca y Mg, respectivamente, y de $172.6,17$ y $29.3 \mathrm{mg} \mathrm{kg}^{-1}$ para Fe, Cu y Zn, con la fertilización mineral; estos valores superan a los que se obtuvieron bajo diferentes dosis de estiércol bovino. Con excepción del Mg, los valores aquí obtenidos también son menores a los reportados por Fernández-Pavía et al. (2015) con solución nutritiva completa, en un estudio sobre inducción de deficiencias en nopal verdura var. Milpa Alta.

El papel que juegan los micronutrientes en la salud humana es esencial; el Zn se encuentra presente en todos los órganos, tejidos, fluidos y secreciones del cuerpo humano. Aproximadamente $83 \%$ del Zn en el cuerpo está presente en el músculo y hueso, y $95 \%$ se encuentra a nivel intracelular y desempeña una función importante en más de 300 enzimas, incluidas las de ADN (Jackson, 1989). Por su parte, el Mn es importante debido a que muchas enzimas dependen de él para producir metaloenzimas, las cuales participan en el uso de la glucosa y en la degradación de proteínas y grasas; se almacena principalmente en los huesos, aunque también se le encuentra en los riñones y el hígado (Grotto, 2014).
El B participa en el mantenimiento y desarrollo adecuado de los huesos, al participar en el metabolismo del calcio, magnesio y fósforo, y se le asocia con la mejora en la respuesta del sistema inmunitario ante determinadas afecciones, lo que es útil para aumentar las defensas (Nielsen, 1997). Según Grotto (2014), mantener una dieta alta en frutas, verduras, frutos secos y granos enteros es la mejor opción para obtener estos nutrientes. De ahí la importancia de consumir nopalitos verdura que además contienen fibra, clorofila, vitamina C, carbohidratos y proteínas, según indican Guzmán-Loayza y Chávez (2007) y Maki-Díaz et al. (2015).

\section{CONCLUSIONES}

El tratamiento de fertilización tuvo un efecto significativo en la concentración de los minerales $\mathrm{Mn}$, Zn y B en los cladodios jóvenes al momento de cosecha, pero no hubo efecto para el resto de nutrimentos. Los mejores tratamientos fueron fertilización mineral para Mn y $B$, y estiércol de bovino más triple 17 para $\mathrm{Zn}$. Por consiguiente, este tratamiento es importante en la aportación de dicho mineral para la nutrición y salud humana. 


\section{BIBLIOGRAFÍA}

AOAC, Association of Official Analytical Chemist (1980) Official Methods of Analysis. $13^{\text {th }}$ ed. Washington, D.C. $100 \mathrm{p}$

Bremner J. M. (1965) Inorganic forms of nitrogen. In: Methods of Soil Analysis. Part. 2. Chemical and Microbiological Properties. A. G. Norman (ed.). American Society of Agronomy. Agronomy Monograph 9.2 pp:1179-1237.

Bolívar-Fernández N. J., M. C. Valencia-Gutiérrez y E. Sauri-Duch (2009) Recursos Fitogenéticos de Origen Tropical: su Contenido de Micronutrientes. Colección Universitaria Serie: Documentos Científicos. Universidad Autónoma de Campeche, México. 178 p.

Bould C. (1970) The nutrition of fruit trees. In: Physiology of Tree Crops. L. C. Luckwill and C. V. Cuttings (eds). Proccedings of a Simposium held at Long Ashton Research Station University of Bristol. 25-28 March 1969. Academic Press. pp:223-234.

Castellanos J. Z., J. X. Uvalle-Bueno y A. Aguilar-Santelises (2000) Manual de Interpretación de Análisis de Suelos y Aguas. $2^{\mathrm{a}}$ ed. Intagri. México. 201 p

FAO/WHO, Food and Agriculture Organization/World Health Organization (2001) Human vitamin and mineral requeriments. Report of a joint FAO/WHO Expert Consultation, FAO, Rome, $303 \mathrm{p}$

Fernández-Pavía Y. L., J. L. García-Cué, A. López-Jiménez y G. Mora-Aguilera (2015) Inducción de deficiencias nutrimentales en nopal verdura Opuntia ficus indica (L). Revista Mexicana de Ciencias Agrícolas 6:1417-1422.

Flores-Valdez C. A. (2003) Producción y comercialización de nopalitos. In: Nopalitos y Tunas. Producción, Comercialización, Poscosecha e Industrialización. J. Corrales-García y C. A. Flores-Valdez (eds). Universidad Autónoma Chapingo. CIESTAAM-Programa Nopal. pp:19-38.

Grotto D. (2014) Lo Mejor que Puedes Comer. Ed. Grijalbo Vital. México. $366 p$

Grusak M. A. and D. DellaPenna (1999) Improving the nutrient composition of plants to enhance human nutrition and health. Annual Review of Plant Physiology and Plant Molecular Biology 50:133161.

Guzmán-Loayza D. y J. Chávez (2007) Estudio bromatológico del cladodio del nopal (Opuntia ficus-indica) para el consumo humano. Revista Sociedad Química Perú 73:41-45

Jackson M. J. (1989) Physiology of zinc: general aspects. In: Zinc in Human Biology. C. F. Mills. (ed.). Springer-Verlag. London. pp:114.

León-Arteta R. y A. Aguilar-Santelises (1987) Materia orgánica In: Aná- lisis Químico para Evaluar la Fertilidad del Suelo. A. Aguilar-Santelises, J. D. Etchevers-Barra y J. Z. Castellanos (eds.). Sociedad Mexicana de la Ciencia del Suelo. México. pp:85-91.

Maki-Díaz G., C. B. Peña-Valdivia, R. García-Nava, M. L. Arévalo-Galarza, G. Calderón-Zavala y S. Anaya-Rosales (2015) Características físicas y químicas de nopal verdura (Opuntia ficus-indica) para exportación y consumo nacional. Agrociencia 49:31-51.

Murillo-Amador B., A. Flores-Hernández, J. L. García-Hernández, R. D. Valdez-Cepeda, N. V. Ávila-Serrano, E. Troyo-Diéguez and F. H. Ruiz-Espinoza (2005) Soil amendment with organic products increases the production of prickly pear cactus as a green vegetable (nopalitos). Journal of the Professional Association for Cactus Development 7:97-109.

Nielsen F. H. (1997) Boron in human and animal nutrition. Plant and Soil 193:199-208

Pimienta-Barrios E. (1990) El Nopal Tunero. Universidad de Guadalajara. Guadalajara, Jal. México. 235 p.

Sáenz-H. C. (1997) Cladodes: a source of dietary fiber. Journal of the Professional Association for Cactus Development 2:117-123

Sáenz-H. C. (2004) Compuestos funcionales y alimentos derivados de Opuntia spp. In: El Nopal: Tópicos de Actualidad. G. Esparza, R. Valdez y S. Méndez (eds.). Universidad Autónoma de Chapingo, México. pp:211-222.

SAS Institute (versión 9.0) (2000) The SAS system for Windows. Release 9.0 SAS Inst. Cary NC. USA.

SIAP, Servicio de Información Agroalimentario y Pesquera (2014) Cierre de la Producción Agrícola por Cultivo. www.siap.gob.mx/ cierre-de-la-produccion-agricola-por-cultivo/. (Enero 2015).

Sloan E. (1994) Top ten trends to watch and work on. Food Technology 7:89-100.

Stintzing F. and R. Carle (2005) Cactus stems (Opuntia spp.): A review on their chemistry, technology and uses. Molecular Food and Nutrition Research 49:174-194

Torres y Torres N. (2014) Comprueban beneficios de nopal en diabéticos y personas con sobrepeso. http://www.jornada.unam.mx/ ultimas/2014/12/22/comprueban-beneficios-del-nopal-en-personas-diabeticas-y-con-sobrepeso-406.html. (Enero 2016).

Zhao F. J. and P. R. Shewry (2011) Recent developments in modifying crops and agronomic practice to improve human health. Food Policy 36:S94-S101.

Zúñiga-Tarango R., I. Orona-Castillo, C. Vázquez, B. Murillo-Amador, E. Salazar-Sosa, J. D. López-Martínez, J. L. García-Hernández and E. Rueda-Puente (2009) Root growth yield and mineral concentration of Opuntia ficus-indica (L.) Mill. under different fertilization treatments. Journal of the Professional Association for Cactus Development 11:53-68. 
